\title{
Celiac Disease is Misdiagnosed Based on Serology Only in a Substantial Proportion of Patients
}

\author{
Stephan R. Vavricka, MD, ${ }^{*} \dagger$ Teresa Stelzer, MD, $\dagger$ Jaqueline Lattmann, MS, $\dagger$ \\ Matthias Stotz, MS, $\dagger$ Romina Lehmann, MS, $\dagger$ Jonas Zeitz, MD,* \\ Michael Scharl, MD,* Benjamin Misselwitz, MD, ${ }^{*}$ Daniel Pohl, MD,* \\ Michael Fried, MD, ${ }^{*}$ Radu Tutuian, MD,\$ Alessio Fasano, MD, $\S$ \\ Alain M. Schoepfer, MD,\| Gerhard Rogler, MD, PhD,* \\ Luc Biedermann, MD,* and Thomas Greuter, MD*
}

\begin{abstract}
Background: Although the diagnostic process in celiac disease (CeD) has been addressed in several international guidelines, little is known about the actual proceeding in current clinical practice. This study investigated the initial presentation, the diagnostic process, follow-up evaluations, and adherence to a gluten-free diet in CeD patients in a real-life setting in Switzerland from a patient's perspective.
\end{abstract}

Methods: We performed a large patient survey among unselected $\mathrm{CeD}$ patients in Switzerland.

Results: A total of 1689 patients were analyzed. The vast majority complained of both gastrointestinal and nonspecific symptoms $(71.5 \%)$, whereas $1.8 \%$ reported an asymptomatic disease course. A total of $35.8 \% \mathrm{CeD}$ patients were diagnosed by a nongastroenterologist. The diagnostic process differed between nongastroenterologists and gastroenterologists, with the latter more often using duodenal biopsy alone or in combination with serology $(94.7 \%$ vs. $63.0 \%)$ and nongastroenterologists more frequently establishing the diagnosis without endoscopy $(37.0 \%$ vs. $5.3 \%$, $P<0.001)$. Follow-up serology after 6 months was performed only in half of all patients $(49.4 \%$ ), whereas $69.9 \%$ had at least 1 followup serology within the first year after diet initiation. About $39.7 \%$ had a follow-up endoscopy with duodenal biopsies (after a median of $12 \mathrm{mo}$; range, 1 to $600 \mathrm{mo}$ ). The likelihood of receiving any

Received for publication July 10, 2016; accepted July 28, 2016.

From the *Division of Gastroenterology and Hepatology, University Hospital Zurich; $\dagger$ Division of Gastroenterology and Hepatology, Triemli Hospital Zurich, Zurich; †Division of Gastroenterology and Hepatology, Spital Tiefenau, Bern; \|Division of Gastroenterology and Hepatology, University Hospital LausanneCHUV, Lausanne, Switzerland; and §Pediatric Gastroenterology and Nutrition, MassGeneral Hospital for Children, Boston, MA.

S.R.V., T.S., and T.G. share co-first authorship.

L.B. and T.G. share co-last authorship.

S.V.R. and T.S. were responsible for the study design, acquisition and analysis of data, and drafting of the manuscript. J.L., M.St., and R.L. were responsible for acquisition and analysis of data. J.Z., M.Sc., B.M., D.P., M.F., R.T., A.F., A.M.S., and G.R. critically revised the manuscript. L.B. and T.G. were responsible for the study design, acquisition and analysis of data, and drafting of the manuscript.

The authors declare that they have nothing to disclose.

Address correspondence to: Stephan R. Vavricka, MD, Division of Gastroenterology and Hepatology, Triemli Hospital Zurich, Birmensdorferstrasse 497, Zurich 8063, Switzerland (e-mail: stephan.vavricka@triemli.zuerich.ch).

Supplemental Digital Content is available for this article. Direct URL citations appear in the printed text and are provided in the HTML and PDF versions of this article on the journal's Website, www.jcge.com.

Copyright (C) 2016 Wolters Kluwer Health, Inc. All rights reserved.

DOI: $10.1097 /$ MCG.0000000000000676 follow-up examination was higher in patients initially diagnosed by a gastroenterologist.

Conclusions: A significant proportion of $\mathrm{CeD}$ patients are diagnosed by nongastroenterologists. Under the diagnostic lead of the latter, more than a third of the patients receive their diagnosis on the basis of a positive serology and/or genetics only, in evident violation of current diagnostic guidelines, which may lead to an overdiagnosis of this entity.

Key Words: celiac disease, diagnostic work-up, serologic testing, duodenal biopsy, gluten-free diet

(J Clin Gastroenterol 2016;00:000-000)

Celia eliac disease (CeD) is a systemic immune-mediated disorder triggered by gluten intake in genetically predisposed patients. ${ }^{1} \mathrm{CeD}$ affects $0.6 \%$ to $1.0 \%$ of the general population worldwide, ${ }^{2-7}$ with a higher prevalence among patients with other autoimmune disorders including type 1 diabetes mellitus or thyroid diseases and genetic disorders such as Turner or Down syndrome. ${ }^{8}$ Regional differences among comparably developed countries and a female preponderance for $\mathrm{CeD}$ have been described; however, the reasons for these remain unclear. ${ }^{9,10}$ Increasing prevalence in developing countries seems to be attributable to a western diet, changes in wheat production, better diagnostics, and increased awareness of the disease. ${ }^{1}$ Serologic screening studies suggest a high number of clinically unrecognized $\mathrm{CeD}$ cases, ${ }^{2,9}$ accordingly, in most patients, CeD remains undiagnosed.

$\mathrm{CeD}$ remains a diagnostic challenge due to the broad range of possible clinical manifestations. In contrast, $\mathrm{CeD}$ symptoms differ according to age at manifestation: infants more often suffer from diarrhea and failure to thrive, ${ }^{11}$ whereas adolescents may complain of extraintestinal manifestations such as a short stature or neurological symptoms. $^{12}$ However, typical gastrointestinal symptoms, among which diarrhea is the most frequently reported, are encountered in only half of all cases. ${ }^{13}$ Furthermore, some $\mathrm{CeD}$ cases are suspected by laboratory findings only such as elevated liver enzymes or otherwise unexplained irondeficiency anemia. Moreover, $\mathrm{CeD}$ may be incidentally diagnosed during upper endoscopies performed for other indications.

Current guidelines recommend serology and duodenal biopsy for $\mathrm{CeD}$ diagnosis with biopsy considered as its mainstay. ${ }^{14}$ Patients with a high clinical suspicion of $\mathrm{CeD}$ 
should undergo endoscopy and duodenal biopsy regardless of the initial serologic testing. As an exception, recently published European pediatric guidelines suggest a place for $\mathrm{CeD}$ diagnosis without the need for duodenal biopsies in the presence of typical symptoms, high serologic titers, and predisposing human leukocyte antigen (HLA) genotypes (DQ2 and/or DQ8). ${ }^{15}$ However, this practice remains highly controversial outside Europe ${ }^{16}$ and among some European pediatric gastroenterologists. ${ }^{17}$ It seems evident that $\mathrm{CeD}$ diagnosis has to be established with high accuracy in every patient considering its pronounced consequences for patients' everyday life: a gluten-free diet (GFD) with life-long elimination of wheat, rye, and barley imposes strong dietary restrictions and has social and economic consequences. In addition, incorrectly diagnosed CeD patients triggers significant health care costs due to unnecessary follow-up visits. Because of the need of a very high diagnostic threshold, clinical improvement after implementation of GFD is not considered sufficient to secure the diagnosis of CeD. ${ }^{18}$ Furthermore, given that histologic findings in $\mathrm{CeD}$ are characteristic, but nonspecific, ${ }^{19}$ some experts even recommend the repetition of duodenal biopsies after 1 year of dietary therapy. ${ }^{14}$ However, how these sometimes conflicting expert recommendations regarding $\mathrm{CeD}$ diagnosis are implemented in clinical practice remains unclear. Close follow-up especially in the first year after diagnosis is recommended for all CeD patients. Assessing adherence to GFD is key and consists of 4 steps: (i) clinical assessment of symptoms, (ii) a dietetic review, (iii) serum antibodies, and (iv) possibly follow-up biopsies, which are recommended if the condition does not respond to GFD or if patients are at an increased risk of lymphoma. ${ }^{14}$ Considering the evidence that adherence to GFD and mucosal healing can prevent $\mathrm{CeD}$ complications, such a thorough follow-up of CeD patients seems essential. ${ }^{20}$ However, compliance with these recommendations in a real-life setting has not been addressed.

This study investigates the initial presentation, the diagnostic process, follow-up evaluations, and adherence to GFD in CeD patients in a real-life setting in Switzerland from a patient's perspective.

\section{MATERIAL AND METHODS}

\section{Study Design}

This large patient survey collected information about CeD presentation, the diagnostic process, treatment, and adherence to GFD on the basis of an exclusive patient perspective. The call to $\mathrm{CeD}$ patients was made through announcements in print news of the Celiac Community Foundation of the German-speaking part of Switzerland and on its website. Patients or their caregivers (for pediatric patients) were prompted to report their own case to the database using a standardized questionnaire. Patient answers and all additional data were anonymized. The study was presented to the local Ethics committee and the need for approval was waived due to anonymized data collection.

\section{Patients and Data Collection}

All patients diagnosed with $\mathrm{CeD}$ were eligible for inclusion in this study. Data were exclusively collected using a questionnaire, which included sections about: (1) patient demographic data, (2) disease characteristics (first symptoms, age at disease onset) and diagnostic process, (3)
GFD and adherence to therapy, (4) disease course and follow-up visits after implementation of dietary restrictions. Clinical remission at follow-up visits was defined as the complete absence of any CeD symptom. For comparison between adult and pediatric study populations, adults were defined as patients above the age of 18 years according to other CeD studies. ${ }^{21}$

\section{Statistical Analysis}

For all statistical analyses, IBM software SPSS version 23.0.0 (2014 SPSS Science Inc., Chicago, IL) was used. Categorical data are summarized as the percentage of the group total. Comparisons between categorical data were performed using the $\chi^{2}$ test or the Fisher exact test in case of a small sample size $(\mathrm{n}<10)$. A 2 -sided $P$-value of $<0.05$ was regarded as statistically significant. For a more detailed subgroup analysis, patients who were younger than 18 years at CeD diagnosis were excluded.

\section{RESULTS}

\section{Patient Demographics}

From a total of 3800 printed questionnaires, 1689 $(44.4 \%)$ were returned and finally analyzed. Demographic data of the study population have been published elsewhere: 1284 participants were female $(76 \%)$, the mean age was 41.3 years (range, 0 to $92 \mathrm{y}$ ) with a mean age at $\mathrm{CeD}$ diagnosis of 31.1 years (range, 0 to $83 \mathrm{y}$ ), ${ }^{22}$ and 449 patients $(26.6 \%)$ were below 18 years at the time of $\mathrm{CeD}$ diagnosis.

\section{First Presentation and Diagnosis of CeD}

Only $15.3 \%$ of the patients reported isolated gastrointestinal symptoms as their initial presenting symptoms. The vast majority complained of both gastrointestinal and nonspecific symptoms $(71.5 \%)$. About $1.8 \%$ reported an asymptomatic disease course. Among all symptoms, flatulence, abdominal pain, and diarrhea were the most frequently reported with at least one of them was present in $>50 \%$ of all patients (Supplementary table 1, Supplemental Digital Content 1, http://links.lww.com/JCG/ A282).

Most patients first presented to a nongastroenterologist $(75.1 \%)$. However, the final diagnosis of $\mathrm{CeD}$ was established in only $35.8 \%$ of the cases by a nongastroenterologist (Table 1). Diagnostic delay by physicians tended to be shorter if patients presented to a gastroenterologist first (mean 31.6 vs. 41.3 months, median 1.0 vs. 5.0 months, $P=0.093$ ). About $16.4 \%$ of all patients were diagnosed on the basis of serology and/or genetics (HLAII DQ2 and DQ8), whereas in the vast majority of the cases, the diagnosis was established by a combination of serology and duodenal biopsy $(46.9 \%)$ or biopsy alone $(31.9 \%)$. In the remaining cases, diagnostic steps were not specified. Under the diagnostic lead of nongastroenterologists, the diagnostic process differed significantly compared with that of gastroenterologists: Gastroenterologists more often used duodenal biopsy alone or in combination with serology (94.7\% vs. $63.0 \%, P<0.001)$, whereas diagnosis was more frequently established without endoscopy by nongastroenterologists $(37.0 \%$ vs. $5.3 \%, P<0.001)$. Differences remained significant if only adult patients $(18 \mathrm{y}$ or above at diagnosis) were analyzed $(95.6 \%$ vs. $58.5 \%$, and $41.5 \%$ vs. $4.4 \%, P<0.001)$. 


\begin{tabular}{|c|c|c|}
\hline Diagnostic Modality & $\begin{array}{c}\text { Diagnosis by a Nongastroenterologist } \\
(\mathrm{N}=\mathbf{3 5 4})(\mathbf{1 0 0 \%})[\mathrm{n}(\%)]\end{array}$ & $\begin{array}{l}\text { Diagnosis by a Gastroenterologist } \\
(\mathrm{N}=\mathbf{7 5 8})(\mathbf{1 0 0 \%})[\mathrm{n}(\%)]\end{array}$ \\
\hline \multicolumn{3}{|l|}{ Adult patients ( $18 \mathrm{y}$ or above at diagnosis) } \\
\hline Serology alone & $126(35.6)$ & $26(3.4)$ \\
\hline EGD and biopsy alone & $51(14.4)$ & $346(45.6)$ \\
\hline Serology and EGD/biopsy & $156(44.1)$ & $379(50.0)$ \\
\hline \multirow{4}{*}{ Others (genetics alone, serology and genetics) } & $21(5.9)$ & $7(0.9 \%)$ \\
\hline & $\begin{array}{c}\text { Genetics (3), genetics and serology (6), } \\
\text { not specified (12) }\end{array}$ & $\begin{array}{c}\text { Genetics (2), genetics and serology (2), } \\
\text { not specified (3) }\end{array}$ \\
\hline & & $P<0.001$ (biopsy vs. nonbiopsy) \\
\hline & $N=592(100 \%)[n(\%)]$ & $N=1006(100 \%)[n(\%)]$ \\
\hline \multicolumn{3}{|l|}{ All patients } \\
\hline Serology alone & $181(30.6)$ & $36(3.6)$ \\
\hline EGD and biopsy alone & 105 (17.7) & $430(42.7)$ \\
\hline Serology and EGD/biopsy & $268(45.3)$ & $523(52.0)$ \\
\hline \multirow{3}{*}{ Others (genetics alone, serology and genetics) } & $38(6.4)$ & $17(1.7)$ \\
\hline & $\begin{array}{c}\text { Genetics (5), genetics and serology (14), } \\
\text { not specified (19) }\end{array}$ & $\begin{array}{c}\text { Genetics (4), genetics and serology (8), } \\
\text { not specified (5) }\end{array}$ \\
\hline & & $P<0.001$ (biopsy vs. nonbiopsy) \\
\hline
\end{tabular}

\section{CeD Treatment and Follow-Up}

GFD remains the mainstay of therapy as $97.7 \%$ of the patients reported having been treated by GFD. More than 3 of 4 patients $(79.8 \%)$ received expert nutrition counseling. This counseling was more often prescribed if $\mathrm{CeD}$ diagnosis was established by a gastroenterologist $(83.5 \%$ vs. $76.0 \%$, $P=0.001)$. About $77.0 \%$ of all patients reported adhering always and $20.7 \%$ with only minor mistakes to a GFD. Patients who were only incidentally diagnosed with CeD tended to adhere to dietary treatment less than patients with symptoms at diagnosis $(63.3 \%$ vs. $77.7 \%, P=0.06)$. No difference regarding adherence to GFD was seen in patients with gastrointestinal symptoms compared with those with a nongastrointestinal presentation. Interestingly, younger patients (aged below $30 \mathrm{y}$ ) reported higher adherence than patients aged over 30 years $(80.5 \%$ vs. $76.2 \%$ for adherence "always" to GFD, $P=0.05$ ).

Follow-up serology after 6 months was performed in half of all patients $(49.4 \%$ ), whereas $69.9 \%$ had at least 1 follow-up serology within the first year after diet initiation. Serology results were infrequently reported; however, at least $19.3 \%$ had a positive serology after 6 months $(53.8 \%$ missing) and $18.5 \%$ after 12 months (41.9\% missing), respectively. About $39.7 \%$ of the patients had a follow-up endoscopy with duodenal biopsies (median duration after diet initiation $12 \mathrm{mo}$; range, 1 to $600 \mathrm{mo}$ ). In adult patients (18y or above). follow-up serologies were more frequently performed if the $\mathrm{CeD}$ diagnosis was established by a gastroenterologist (at $6 \mathrm{mo} 58.7 \%$ vs. $51.2 \%, P=0.032$, at 12 mo $62.6 \%$ vs. $51.6 \%, P=0.002$ ). In addition, follow-up biopsies were more often performed in adult patients diagnosed by a gastroenterologist compared with those who were initially diagnosed by a nongastroenterologist (54.0\% vs. $44.9 \%, P=0.007$ ) (for details, see Figure 1).

About $43.7 \%$ of the patients reported complete clinical remission 6 months after GFD initiation. The proportion of complete remission was $59.4 \%$ after 12 months compared with $61.7 \%$ after 24 months. No difference regarding clinical remission was seen in those patients initially diagnosed by a gastroenterologist compared with those diagnosed by a nongastroenterologist, either at 6 months or at 12 and 24 months after $\mathrm{CeD}$ diagnosis. Similar results were seen if only adult patients were analyzed.

\section{DISCUSSION}

In this large patient survey, we report the presentation, diagnostic work-up, treatment by GFD, and follow-up evaluations in $\mathrm{CeD}$ from a patient's perspective. In contrast to actual $\mathrm{CeD}$ guidelines, up to one sixth of all $\mathrm{CeD}$ patients were diagnosed by serology only: reaching even 4 of 10 patients if diagnosis was established by nongastroenterologists. Furthermore, we demonstrate a high frequency of nonspecific, nonclassical $\mathrm{CeD}$ symptoms and a mismatch between self-reported diet adherence and clinical remission rates.

In accordance to Rampertab et $\mathrm{al}^{13}$ and other studies evaluating the presentation of $\mathrm{CeD},{ }^{11}$ diarrhea and abdominal pain are among the most frequently reported CeD symptoms. However, $>40 \%$ of our patients did not suffer from diarrhea, which is believed to be the classical $\mathrm{CeD}$ symptom. In addition, nonclassical gastrointestinal

\section{Follow-up in adult patients}

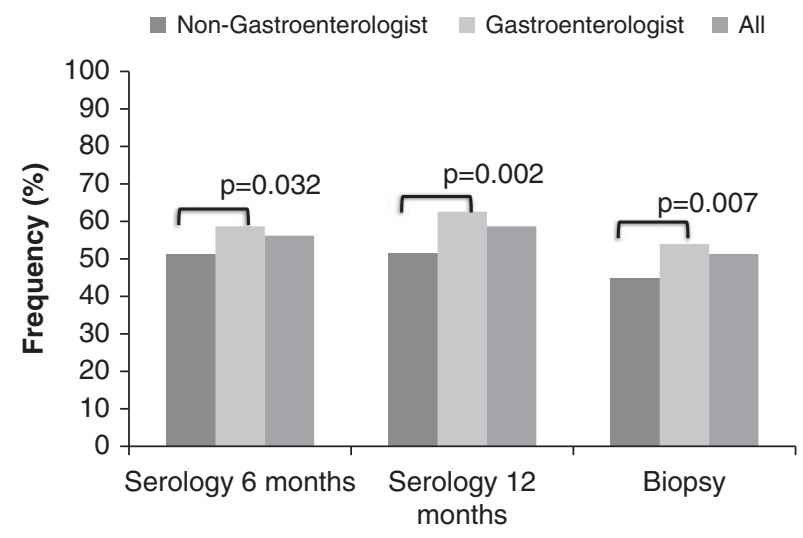

FIGURE 1. Follow-up evaluations in adult patients after diet initiation. 
symptoms such as flatulence, constipation, nausea, or vomiting are frequently encountered. Only $15 \%$ presented with isolated gastrointestinal symptoms, underlining the crucial importance of awareness toward nonspecific CeD presentations such as fatigue or unexplained weight loss. Exceptional symptoms such as edema, skin lesions, migraine, or even paresthesia are not uncommon in our cohort; up to 1 of 10 patients presented with one of these symptoms. In any case, in our cohort, most patients recalled at least some presenting symptoms and an asymptomatic disease course was reported only by $2 \%$ patients. This number contrasts prior findings, for instance by Rutz et al, ${ }^{23}$ suggesting a higher prevalence of asymptomatic CeD compared with symptomatic forms. Given the high frequency of nonspecific gastrointestinal and nongastrointestinal symptoms, CeD should also be considered in the absence of a classical presentation with diarrhea and abdominal pain. CeD screening in these patients will possibly uncover more $\mathrm{CeD}$ cases as seen in the study of Catassi et al. ${ }^{24}$

Importantly, we found that up to one sixth of all CeD patients received their diagnosis without endoscopic assessment on the basis of serology and/or genetics only. This apparent clinical practice contrasts current CeD diagnostic guidelines, ${ }^{14}$ which consider upper endoscopy with duodenal biopsy as the mainstay in $\mathrm{CeD}$ diagnosis. Furthermore, we demonstrate that CeD without endoscopy was significantly more often diagnosed by nongastroenterologists. Almost 4 of 10 nongastroenterologists made a CeD diagnosis on the basis of serology and concomitant symptoms/signs only. If we further take into account that up to one third of the patients received their diagnosis by a nongastroenterologist, then in more than 200 patients $(12 \%) \mathrm{CeD}$ diagnosis was not established in accordance to diagnostic guidelines. In addition to that, even gastroenterologists did not follow diagnostic guidelines rigourously. In summary, $>16 \%$ of all 1689 patients did not receive their diagnosis correctly. Biagi et $\mathrm{al}^{25}$ have recently shown that in 61 of 180 patients who did not receive their $\mathrm{CeD}$ diagnosis correctly, the diagnosis of $\mathrm{CeD}$ could not be confirmed after reinvestigation. Thus, there may indeed be a substantial risk of overdiagnoses of the $\mathrm{CeD}$ entity and therefore unnecessary, lifelong dietary restrictions and follow-up evaluations. This finding has important clinical implications as implementing an accurate diagnostic process ${ }^{14}$ might not only impact on individual patient's life but also safe health care costs.

The reported excellent adherence rate to GFD is $>70 \%$ in our cohort. Our results agree with previous findings, although our adherence rates are definitely in the upper limit. In addition, self-reported adherence rates are notoriously higher than true adherence rates. ${ }^{26}$ In the metaanalysis of Hall et al, ${ }^{27}$ strict adherence rates measured by self-report ranged from $42 \%$ to $91 \%$. In contrast to other studies, where the effect of age is unclear ${ }^{28,29}$ or younger age even correlated with lower adherence, ${ }^{30,31}$ we found that adult patients under 30 years of age show better adherence. In addition, symptomatic $\mathrm{CeD}$ patients tend to adhere better than those with an asymptomatic disease course, also contrasting prior findings. ${ }^{27}$ In our cohort, high adherence rates to GFD contrast with the relatively lower rates of clinical remission of $50 \%$ at 6 months after diagnosis, although some increase was observed over the following 2 years. This mismatch may certainly be explained by a self-report assessment and therefore a possible misinterpretation of adherence to GFD by each individual patient, despite a relatively high proportion of patients receiving specialized nutrition counseling. Of note, such counseling was more often prescribed, if a gastroenterologist was involved in the $\mathrm{CeD}$ diagnosis and followup evaluations. In addition, follow-up examinations (serology, biopsy) were more often ordered/performed by gastroenterologists. However, both gastroenterologists and nongastroenterologists did not use them as recommended in expert guidelines. It remains unclear whether the more rigorous but imperfect follow-up by gastroenterologists impacts the clinical course of patients because neither adherence rates to GFD nor rates of clinical remission were higher in patients diagnosed and treated by a gastroenterologist compared with a nongastroenterologist.

Our study has several limitations: (i) exclusively relying on self-reports may have led to an overestimation of adherence rates. However, these rates are comparable to those in other studies ${ }^{27}$ and verifying true adherence remains a challenge regardless of assessment by a physician. (ii) Because of the retrospective assessment of symptoms, our results are prone to recall bias. (iii) Another limitation may be the absence of external validation including physician assessment or chart review. Misperception of diagnostic steps may have led to an overestimation of CeD cases diagnosed without endoscopy and duodenal biopsy. However, the rates of missing values regarding the $\mathrm{CeD}$ diagnostic process are low [78/1689 (4.6\%) in all patients; $6 /$ $1124(3.2 \%)$ in adult patients], supporting an adequate patient perception. (iv) Another limitation is a possible selection bias due to exclusive inclusion of patients who were members of the Celiac Community Foundation of the German-speaking part of Switzerland. Patients with a symptomatic CeD course may more likely be members of this foundation, which is supported by the fact that asymptomatic $\mathrm{CeD}$ patients are underrepresented in this study.

In conclusion, more than 1 of $3 \mathrm{CeD}$ patients are diagnosed by nongastroenterologists. Under the diagnostic lead of nongastroenterologists, more than a third of the patients receive their diagnosis on the basis of a positive serology and/or genetics only, in evident violation of current diagnostic guidelines. ${ }^{14}$ This may lead to a substantial proportion of overdiagnoses and therefore unnecessary, potentially lifelong dietary restrictions as well as follow-up evaluations in these patients. In addition, nonspecific nonclassical symptoms - either gastrointestinal or nongastrointestinal-are frequent, and lack of diarrhea and/or abdominal pain should not delay diagnostic work-up.

\section{REFERENCES}

1. Fasano A, Catassi C. Clinical practice. Celiac disease. $N$ Engl J Med. 2012;367:2419-2426.

2. West J, Logan RF, Hill PG, et al. Seroprevalence, correlates, and characteristics of undetected coeliac disease in England. Gut. 2003;52:960-965.

3. Mäki M, Mustalahti K, Kokkonen J, et al. Prevalence of celiac disease among children in Finland. $N$ Engl $J$ Med. 2003;348:2517-2524.

4. Tatar G, Elsurer R, Simsek H, et al. Screening of tissue transglutaminase antibody in healthy blood donors for celiac disease screening in the Turkish population. Dig Dis Sci. 2004;49:1479-1484.

5. Fasano A, Berti I, Gerarduzzi T, et al. Prevalence of celiac disease in at-risk and not-at-risk groups in the United States: a large multicenter study. Arch Intern Med. 2003;163:286-292. 
6. Bingley PJ, Williams AJ, Norcross AJ, et al. Undiagnosed coeliac disease at age seven: population based prospective birth cohort study. BMJ. 2004;328:322-323.

7. Biagi F, Klersy C, Balduzzi D, et al. Are we not overestimating the prevalence of coeliac disease in the general population? Ann Med. 2010;42:557-561.

8. American Gastroenterological Association Institute. AGA institute medical position statement on the diagnosis and management of celiac disease. Gastroenterology. 2006;131:1977-1980.

9. Mustalahti K, Catassi C, Reunanen A, et al. The prevalence of celiac disease in Europe: results of a centralized, international mass screening project. Ann Med. 2010;42:587-595.

10. Green PHR, Stavropoulos SN, Panagi SG, et al. Characteristics of adult celiac disease in the USA: results of a national survey. Am J Gastroenterol. 2001;96:126-131.

11. Green PH, Cellier C. Celiac disease. N Engl J Med. 2007;357: 1731-1743.

12. D'Amico MA, Holmes J, Stavropoulos SN, et al. Presentation of pediatric celiac disease in the United States: prominent effect of breastfeeding. Clin Pediatr (Phila). 2005;44:249-258.

13. Rampertab SD, Pooran N, Brar $\mathrm{P}$, et al. Trends in the presentation of celiac disease. Am J Med. 2006;119:355. e9-14.

14. Ludvigsson JF, Bai JC, Biagi F, et al. Diagnosis and management of adult coeliac disease: guidelines from the British Society of Gastroenterology. Gut. 2014;63:1210-1228.

15. Husby S, Koletzko S, Korponay-Szabó IR, et al. European Society for Pediatric Gastroenterology, Hepatology, and Nutrition guidelines for the diagnosis of coeliac disease. $J$ Pediatr Gastroenterol Nutr. 2012;54:136-160.

16. Hill ID, Dirks MH, Liptak GS, et al. Guideline for the diagnosis and treatment of celiac disease in children: recommendations of the North American Society for Pediatric Gastroenterology, Hepatology and Nutrition. $J$ Pediatr Gastroenterol Nutr. 2005;40:1-19.

17. Schirru E, Jores RD, Congia M. Prudence is necessary in the application of the new ESPGHAN criteria for celiac disease omitting duodenal biopsy: a case report. Eur J Gastroenterol Hepatol. 2014;26:679-680.

18. Biesiekierski JR, Peters SL, Newnham ED, et al. No effects of gluten in patients with self-reported non-celiac gluten sensitivity after dietary reduction of fermentable, poorly absorbed, short-chain carbohydrates. Gastroenterology. 2013;145:320-328. e321-e323.
19. Memeo L, Jhang J, Hibshoosh H, et al. Duodenal intraepithelial lymphocytosis with normal villous architecture: common occurrence in H. pylori gastritis. Mod Pathol. 2005;18:1134-1144.

20. Haines ML, Anderson RP, Gibson PR. Systematic review: the evidence base for long-term management of coeliac disease. Aliment Pharmacol Ther. 2008;28:1042-1066.

21. Kurppa K, Paavola A, Collin P, et al. Benefits of a gluten-free diet for asymptomatic patients with serologic markers of celiac disease. Gastroenterology. 2014;147:610-617, e611.

22. Vavricka SR, Vadasz N, Stotz M, et al. Celiac disease diagnosis still significantly delayed - doctor's but not patients' delay responsive for the increased total delay in women. Dig Liver Dis. 2016. [Epub ahead of print]. S1590-8658(16)30475-3.

23. Rutz R, Ritzler E, Fierz W, et al. Prevalence of asymptomatic celiac disease in adolescents of eastern Switzerland. Swiss Med Wkly. 2002;132:43-47.

24. Catassi C, Kryszak D, Louis-Jacques O, et al. Detection of celiac disease in primary care: a multicenter case-finding study in North America. Am J Gastroenterol. 2007;102: 1454-1460.

25. Biagi F, Bianchi PI, Campanella $\mathrm{J}$, et al. The impact of misdiagnosing celiac disease at a referral centre. Can $J$ Gastroenterol. 2009;23:543-545.

26. Oxentenko AS, Murray JA. Celiac disease: ten things that every gastroenterologist should know. Clin Gastroenterol Hepatol. 2015;13:1396-1404.

27. Hall NJ, Rubin G, Charnock A. Systematic review: adherence to a gluten-free diet in adult patients with coeliac disease. Aliment Pharmacol Ther. 2009;30:315-330.

28. Leffler DA, Edwards-George J, Dennis M, et al. Factors that influence adherence to a gluten-free diet in adults with celiac disease. Dig Dis Sci. 2008;53:1573-1581.

29. Viljamaa M, Collin P, Huhtala $\mathrm{H}$, et al. Is coeliac disease screening in risk groups justified? A fourteen-year follow-up with special focus on compliance and quality of life. Aliment Pharmacol Ther. 2005;22:317-324.

30. Ciacci C, Cirillo M, Cavallaro R, et al. Long-term follow-up of celiac adults on gluten-free diet: prevalence and correlates of intestinal damage. Digestion. 2002;66:178-185.

31. Ciacci C, D'Agate C, De Rosa A, et al. Self-rated quality of life in celiac disease. Dig Dis Sci. 2003;48:2216-2220.

Copyright (C) 2016 Wolters Kluwer Health, Inc. Unauthorized reproduction of this article is prohibited.

This paper can be cited using the date of access and the unique DOI number which can be found in the footnotes. 